\title{
Structures and electronic properties of aluminum nanowires
}

AUTHOR(S):

Makita, T; Doi, K; Nakamura, K; Tachibana, A

\section{CITATION:}

Makita, T ... [et al]. Structures and electronic properties of aluminum nanowires. JOURNAL OF CHEMICAL PHYSICS 2003, 119(1): 538-546

\section{ISSUE DATE:}

2003-07-01

URL:

http://hdl.handle.net/2433/39751

\section{RIGHT:}

Copyright 2003 American Institute of Physics. This article may be downloaded for personal use only. Any other use requires prior permission of the author and the American Institute of Physics. 


\title{
Structures and electronic properties of aluminum nanowires
}

\author{
Toshiki Makita, Kentaro Doi, Koichi Nakamura, and Akitomo Tachibana ${ }^{\text {a) }}$ \\ Department of Engineering Physics and Mechanics, Kyoto University, Kyoto 606-8501, Japan
}

(Received 22 March 2002; accepted 25 February 2003)

\begin{abstract}
We report first-principle electronic-state calculations for aluminum (Al) nanowires, which receive much attention as a new device material. First-principle calculations of the periodic boundary nanowire models have been carried out by means of supercell approximation techniques with density functional theory. The stable structures of Al nanowires have been investigated by geometry optimization of model structures of various types. For each stable structure, we have discussed the properties of nanowire in detail, such as radius of wire, interatomic distances, and stability for deformation. Furthermore, the growth and the reconstruction of nanowire by adsoprtion of $\mathrm{Al}$ atom have been simulated. We have also discussed the electronic processes and the driving forces in the growing process of $\mathrm{Al}$ nanowire, in terms of the quantum mechanical energy densities based on the regional density functional theory [Tachibana, J. Chem. Phys. 115, 3497 (2001)]. They have disclosed the driving force of the stability in electronic processes and give new images of microscopic electronic stresses. (C) 2003 American Institute of Physics. [DOI: 10.1063/1.1568086]
\end{abstract}

\section{INTRODUCTION}

Nanoscale materials have peculiar properties different from crystal structures. Currently various types of nanodevices are being studied, and especially metallic nanowires receive a lot of attention in both of industrial and academic fields.

Kondo and Takayanagi succeeded in making suspended gold nanowires in ultra-high vacuum and showed that their finest structure was $0.6 \mathrm{~nm}$ in diameter and $6 \mathrm{~nm}$ in length. They have also revealed their structures consist of coaxial multi-shell tubes by using high-resolution electron microscopy. ${ }^{1}$ Rodrigues et al. determined the structure of gold nanowires generated by mechanical stretching, and estimated quantized conductance just before the nanowires were ruptured. ${ }^{2}$ Nakamura $e t$ al. studied the deformation and breaking of a nanoscale $\mathrm{Na}$ wire by using density functional simulations, and discussed their atomic reconstruction, force, and conductance during elongation. They displayed the cor- relation between atomic reconstruction and quantized conductance. ${ }^{3}$ Gülseren et al. carried out the calculation about $\mathrm{Al}$ nanowires by using the empirical potentials. They made it clear that the structure was changed as the radius got small. They also stated that $a b$ initio would be needed for more precise calculations. ${ }^{4}$

In this article, we have carried out first-principle electronic-state calculations of various types of $\mathrm{Al}$ nanowires, which have geometrical symmetries such as coaxial, helical, and so on. In addition we simulated the process of a pentagonal coaxial nanowire adsorbing an $\mathrm{Al}$ atom and discussed its growth to the hexagonal coaxial structure.

We have also discussed the electronic processes and the driving forces in the radial growth and the reconstruction of Al nanowire, in terms of the quantum mechanical energy densities $^{5-8}$ based on the regional density functional theory. ${ }^{5-12}$

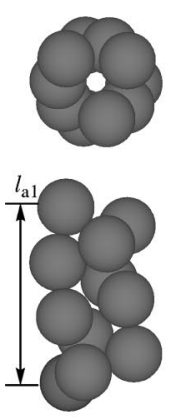

a1
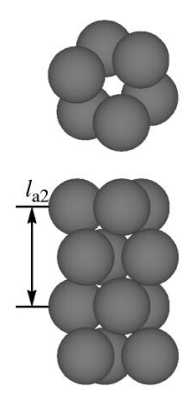

a2

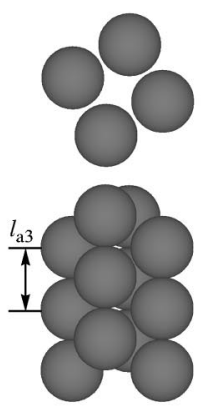

a3

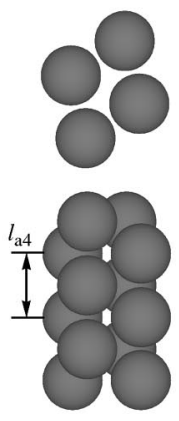

a4

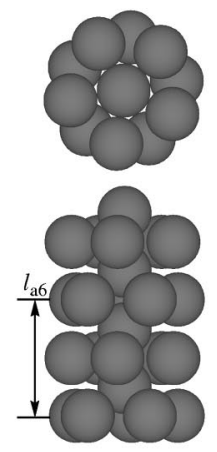

a6

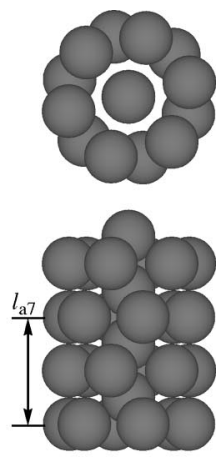

a7

FIG. 1. Top and side views of Al nanowire models.

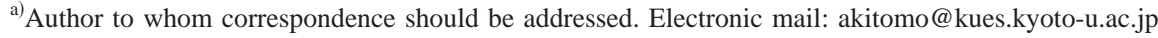


TABLE I. Coordinates of $\mathrm{Al}$ atoms with structural parameters for nanowire models.

\begin{tabular}{|c|c|c|c|c|c|c|c|}
\hline & \multicolumn{3}{|c|}{ a1 } & & \multicolumn{3}{|c|}{$\mathbf{a} 2$} \\
\hline & $x$ & $y$ & $z$ & & $x$ & $y$ & $z$ \\
\hline 1 & $r_{\mathrm{a} 1} \cos 0^{\circ}$ & $r_{\mathrm{a} 1} \sin 0^{\circ}$ & 0 & 1 & $r_{\mathrm{a} 2} \cos 0^{\circ}$ & $r_{\mathrm{a} 2} \sin 0^{\circ}$ & 0 \\
\hline 2 & $r_{\mathrm{a} 1} \cos 108^{\circ}$ & $r_{\mathrm{a} 1} \sin 108^{\circ}$ & $t_{\mathrm{a} 1}$ & 2 & $r_{\mathrm{a} 2} \cos 120^{\circ}$ & $r_{\mathrm{a} 2} \sin 120^{\circ}$ & 0 \\
\hline 3 & $r_{\mathrm{a} 1} \cos 216^{\circ}$ & $r_{\mathrm{a} 1} \sin 216^{\circ}$ & $2 t_{\mathrm{a} 1}$ & 3 & $r_{\mathrm{a} 2} \cos 240^{\circ}$ & $r_{\mathrm{a} 2} \sin 240^{\circ}$ & 0 \\
\hline 4 & $r_{\mathrm{a} 1} \cos 324^{\circ}$ & $r_{\mathrm{a} 1} \sin 324^{\circ}$ & $3 t_{\mathrm{a} 1}$ & 4 & $r_{\mathrm{a} 2} \cos 60^{\circ}$ & $r_{\mathrm{a} 2} \sin 60^{\circ}$ & $t_{\mathrm{a} 2}$ \\
\hline 5 & $r_{\mathrm{a} 1} \cos 432^{\circ}$ & $r_{\mathrm{a} 1} \sin 432^{\circ}$ & $4 t_{\mathrm{a} 1}$ & 5 & $r_{\mathrm{a} 2} \cos 180^{\circ}$ & $r_{\mathrm{a} 2} \sin 180^{\circ}$ & $t_{\mathrm{a} 2}$ \\
\hline 6 & $r_{\mathrm{a} 1} \cos 540^{\circ}$ & $r_{\mathrm{a} 1} \sin 540^{\circ}$ & $5 t_{\mathrm{a} 1}$ & 6 & $r_{\mathrm{a} 2} \cos 300^{\circ}$ & $r_{\mathrm{a} 2} \sin 300^{\circ}$ & $t_{\mathrm{a} 2}$ \\
\hline 7 & $r_{\mathrm{a} 1} \cos 648^{\circ}$ & $r_{\mathrm{a} 1} \sin 648^{\circ}$ & $6 t_{\mathrm{a} 1}$ & & & & \\
\hline 8 & $r_{\mathrm{a} 1} \cos 756^{\circ}$ & $r_{\mathrm{a} 1} \sin 756^{\circ}$ & $7 t_{\mathrm{a} 1}$ & & & & \\
\hline 9 & $r_{\mathrm{a} 1} \cos 864^{\circ}$ & $r_{\mathrm{a} 1} \sin 864^{\circ}$ & $8 t_{\mathrm{a} 1}$ & & & & \\
\hline \multirow[t]{2}{*}{10} & $r_{\mathrm{a} 1} \cos 972^{\circ}$ & $r_{\mathrm{a} 1} \sin 972^{\circ}$ & $9 t_{\mathrm{a} 1}$ & & & & \\
\hline & \multicolumn{3}{|c|}{$\mathbf{a 3}$} & & \multicolumn{3}{|c|}{ a4 } \\
\hline 1 & $r_{\mathrm{a} 3} \cos 0^{\circ}$ & $r_{\mathrm{a} 3} \sin 0^{\circ}$ & 0 & 1 & $r_{\mathrm{a} 4} \cos 0^{\circ}$ & $r_{\mathrm{a} 4} \sin 0^{\circ}$ & 0 \\
\hline 2 & $r_{\mathrm{a} 3} \cos 180^{\circ}$ & $r_{\mathrm{a} 3} \sin 180^{\circ}$ & 0 & 2 & $r_{\mathrm{a} 4} \cos 180^{\circ}$ & $r_{\mathrm{a} 4} \sin 180^{\circ}$ & 0 \\
\hline 3 & $r_{\mathrm{a} 3} \cos 90^{\circ}$ & $r_{\mathrm{a} 3} \sin 90^{\circ}$ & $t_{\mathrm{a} 3}$ & 3 & $r_{\mathrm{a} 4} \cos 90^{\circ}$ & $r_{\mathrm{a} 4} \sin 90^{\circ}$ & $t_{\mathrm{a} 4}$ \\
\hline \multirow[t]{2}{*}{4} & $r_{\mathrm{a} 3} \cos 270^{\circ}$ & $r_{\mathrm{a} 3} \sin 270^{\circ}$ & $t_{\mathrm{a} 3}$ & 4 & $r_{\mathrm{a} 4} \cos 270^{\circ}$ & $r_{\mathrm{a} 4} \sin 270^{\circ}$ & $t_{\mathrm{a} 4}$ \\
\hline & \multicolumn{3}{|c|}{ a6 } & & \multicolumn{3}{|c|}{ a7 } \\
\hline 1 & $r_{\mathrm{a} 6} \cos 0^{\circ}$ & $r_{\mathrm{a} 6} \sin 0^{\circ}$ & 0 & 1 & $r_{\mathrm{a} 7} \cos 0^{\circ}$ & $r_{\mathrm{a} 7} \sin 0^{\circ}$ & 0 \\
\hline 2 & $r_{\mathrm{a} 6} \cos 72^{\circ}$ & $r_{\mathrm{a} 6} \sin 72^{\circ}$ & 0 & 2 & $r_{\mathrm{a} 7} \cos 60^{\circ}$ & $r_{\mathrm{a} 7} \sin 60^{\circ}$ & 0 \\
\hline 3 & $r_{\mathrm{a} 6} \cos 144^{\circ}$ & $r_{\mathrm{a} 6} \sin 144^{\circ}$ & 0 & 3 & $r_{\mathrm{a} 7} \cos 120^{\circ}$ & $r_{\mathrm{a} 7} \sin 120^{\circ}$ & 0 \\
\hline 4 & $r_{\mathrm{a} 6} \cos 216^{\circ}$ & $r_{\mathrm{a} 6} \sin 216^{\circ}$ & 0 & 4 & $r_{\mathrm{a} 7} \cos 180^{\circ}$ & $r_{\mathrm{a} 7} \sin 180^{\circ}$ & 0 \\
\hline 5 & $r_{\mathrm{a} 6} \cos 288^{\circ}$ & $r_{\mathrm{a} 6} \sin 288^{\circ}$ & 0 & 5 & $r_{\mathrm{a} 7} \cos 240^{\circ}$ & $r_{\mathrm{a} 7} \sin 240^{\circ}$ & 0 \\
\hline 6 & 0 & 0 & $t_{\mathrm{a} 6}$ & 6 & $r_{\mathrm{a} 7} \cos 300^{\circ}$ & $r_{\mathrm{a} 7} \sin 300^{\circ}$ & 0 \\
\hline 7 & $r_{\mathrm{a} 6} \cos 36^{\circ}$ & $r_{\mathrm{a} 6} \sin 36^{\circ}$ & $2 t_{\mathrm{a} 6}$ & 7 & 0 & 0 & $t_{\mathrm{a} 7}$ \\
\hline 8 & $r_{\mathrm{a} 6} \cos 108^{\circ}$ & $r_{\mathrm{a} 6} \sin 108^{\circ}$ & $2 t_{\mathrm{a} 6}$ & 8 & $r_{\mathrm{a} 7} \cos 30^{\circ}$ & $r_{\mathrm{a} 7} \sin 30^{\circ}$ & $2 t_{\mathrm{a} 7}$ \\
\hline 9 & $r_{\mathrm{a} 6} \cos 180^{\circ}$ & $r_{\mathrm{a} 6} \sin 180^{\circ}$ & $2 t_{\mathrm{a} 6}$ & 9 & $r_{\mathrm{a} 7} \cos 90^{\circ}$ & $r_{\mathrm{a} 7} \sin 90^{\circ}$ & $2 t_{\mathrm{a} 7}$ \\
\hline 10 & $r_{\mathrm{a} 6} \cos 252^{\circ}$ & $r_{\mathrm{a} 6} \sin 252^{\circ}$ & $2 t_{\mathrm{a} 6}$ & 10 & $r_{\mathrm{a} 7} \cos 150^{\circ}$ & $r_{\mathrm{a} 7} \sin 150^{\circ}$ & $2 t_{\mathrm{a} 7}$ \\
\hline 11 & $r_{\mathrm{a} 6} \cos 324^{\circ}$ & $r_{\mathrm{a} 6} \sin 324^{\circ}$ & $2 t_{\mathrm{a} 6}$ & 11 & $r_{\mathrm{a} 7} \cos 210^{\circ}$ & $r_{\mathrm{a} 7} \sin 210^{\circ}$ & $2 t_{\mathrm{a} 7}$ \\
\hline \multirow[t]{3}{*}{12} & 0 & 0 & $3 t_{\mathrm{a} 6}$ & 12 & $r_{\mathrm{a} 7} \cos 270^{\circ}$ & $r_{\mathrm{a} 7} \sin 270^{\circ}$ & $2 t_{\mathrm{a} 7}$ \\
\hline & & & & 13 & $r_{\mathrm{a} 7} \cos 330^{\circ}$ & $r_{\mathrm{a} 7} \sin 330^{\circ}$ & $2 t_{\mathrm{a} 7}$ \\
\hline & & & & 14 & 0 & 0 & $3 t_{\mathrm{a} 7}$ \\
\hline
\end{tabular}

\section{METHODS OF CALCULATION}

We have carried out first-principle calculations of the periodic boundary nanowire models with the Periodic Regional DFT program package. ${ }^{13}$ We adopted supercell approximation techniques with norm-conserving pseudopotentials prepared according to the Hamann method. ${ }^{14}$ The generalized-gradient approximation (GGA) method by the Perdew-Wang 1991 (PW91) functional was used for density functional theory exchange-correlation interaction. ${ }^{15,16}$ The cutoff energy for wave functions with plane-wave expansion was set at 17 Rydberg $(231.3 \mathrm{eV})$.

\section{RESULTS AND DISCUSSION}

\section{A. Structures of Al nanowires}

Figure 1 shows six models of $\mathrm{Al}$ nanowires which we have devised. The outline of structures and symbols (a1-a4, a6, a7) are referred to Ref. 4. The model a1 has a helical structure, where an angle of rotation for axis is set to $108^{\circ}$ per an atom. In this structure, the coordinate of $n$th $\mathbf{a 1}$ atom can be determined as $\left(r_{\mathrm{a} 1} \cos (n-1) 108^{\circ}, r_{\mathrm{a} 1} \sin (n-1) 108^{\circ}\right.$, $\left.(n-1) t_{\mathrm{a} 1}\right)$ by constant wire radius $r_{\mathrm{a} 1}$ and interlayer distance $t_{\mathrm{a} 1}$.
The model $\mathbf{a} 2$ consists of regular triangle layers with constant wire radius $r_{\mathrm{a} 2}$ piled with constant interlayer distance $t_{\mathrm{a} 2}$ on alternate directions. The structures of $\mathbf{a} 3$ and $\mathbf{a} 4$ are almost equivalent to a fragment of $b c c$ and $f c c$ struc-

TABLE II. Radius of wire $r_{\mathrm{a} i}$, interlayer distance $t_{\mathrm{a} i}$, cell parameter $l_{\mathrm{a} i}$, and interatomic distances in optimized nanowire models.

\begin{tabular}{|c|c|c|c|c|c|}
\hline Model & $r_{\mathrm{a} i}(\AA)$ & $r_{\mathrm{a} i}^{\prime}(\AA)$ & $t_{\mathrm{a} i}(\AA)$ & $l_{\mathrm{a} i}(\AA)$ & $\begin{array}{c}\text { Interatomic } \\
\text { distances }^{\mathrm{a}}(\AA)\end{array}$ \\
\hline a1 & 1.628 & & 0.787 & 7.874 & $\begin{array}{ll}d_{14} & 2.568 \\
d_{12} & 2.750\end{array}$ \\
\hline a2 & 1.653 & & 2.151 & 4.302 & $\begin{array}{ll}d_{12} & 2.713 \\
d_{14} & 2.864\end{array}$ \\
\hline a3 & 1.847 & & 1.217 & 2.434 & $\begin{array}{ll}d_{11}, & 2.434 \\
d_{13} & 2.882\end{array}$ \\
\hline a4 & 2.126 & 1.504 & 1.290 & 2.580 & $\begin{array}{ll}d_{11} & 2.580 \\
d_{13} & 2.906 \\
d_{34} & 3.007\end{array}$ \\
\hline a6 & 2.485 & & 1.330 & 5.359 & $\begin{array}{ll}d_{16} & 2.823 \\
d_{12} & 2.921 \\
d_{17} & 3.089\end{array}$ \\
\hline a7 & 2.864 & & 1.237 & 4.940 & $\begin{array}{ll}d_{12} & 2.864 \\
d_{18} & 2.885 \\
d_{17} & 3.120\end{array}$ \\
\hline
\end{tabular}

${ }^{a} d_{j k}$ means the distance between atoms $j$ and $k$ referred to Table I. Prime of atom index denotes the atom in the next cell. 

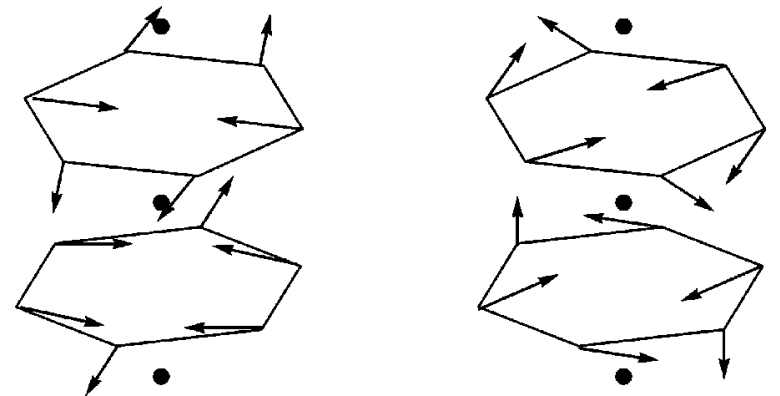

FIG. 2. Example of normal vibrational modes for a7. These are degenerate and the last to show the stability against the deformation along the mode, as the unit cell is enlarged to $14.817 \times 14.817 \times 4.940 \AA^{3}$. Hexagons and dots indicate the $\mathrm{Al}$ atoms.

tures, respectively. For both models, each layer is built by two $\mathrm{Al}$ atoms which cross at a right angle with the next layer. Strains due to extraction from $b c c$ and $f c c$ structures were reflected by constant wire radius $r_{\mathrm{a} 3}$ and interlayer distance $t_{\mathrm{a} 3}$ for $\mathbf{a} 3$, and constant major radius $r_{\mathrm{a} 4}$, minor radius $r_{\mathrm{a} 4}^{\prime}$ and interlayer distance $t_{\mathrm{a} 4}$ for $\mathbf{a} 4$, respectively.

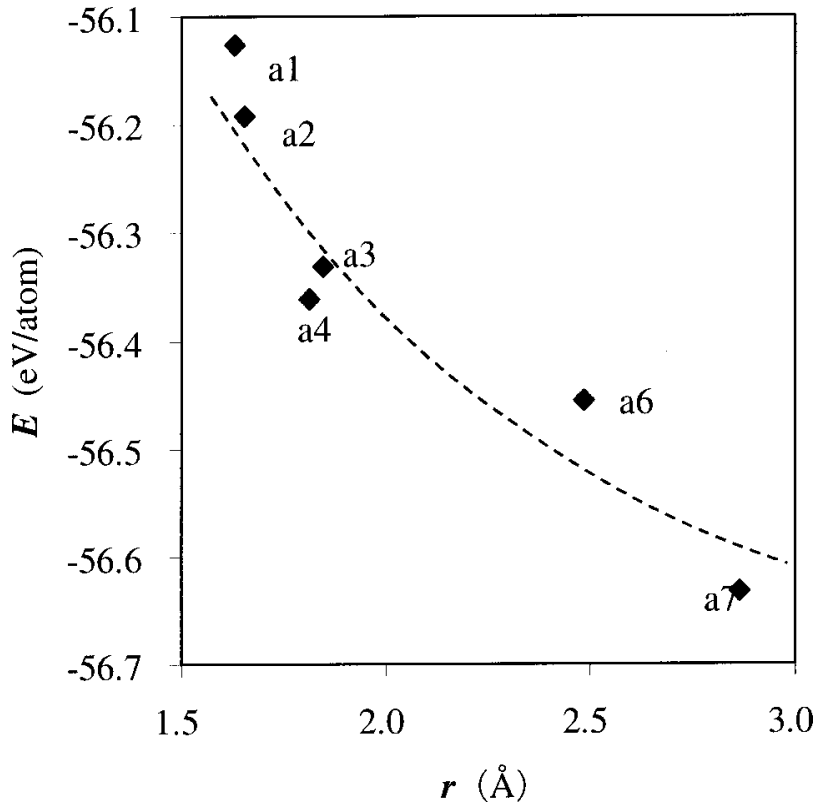

FIG. 3. Energy per atom with respect to the wire radius of each structure.
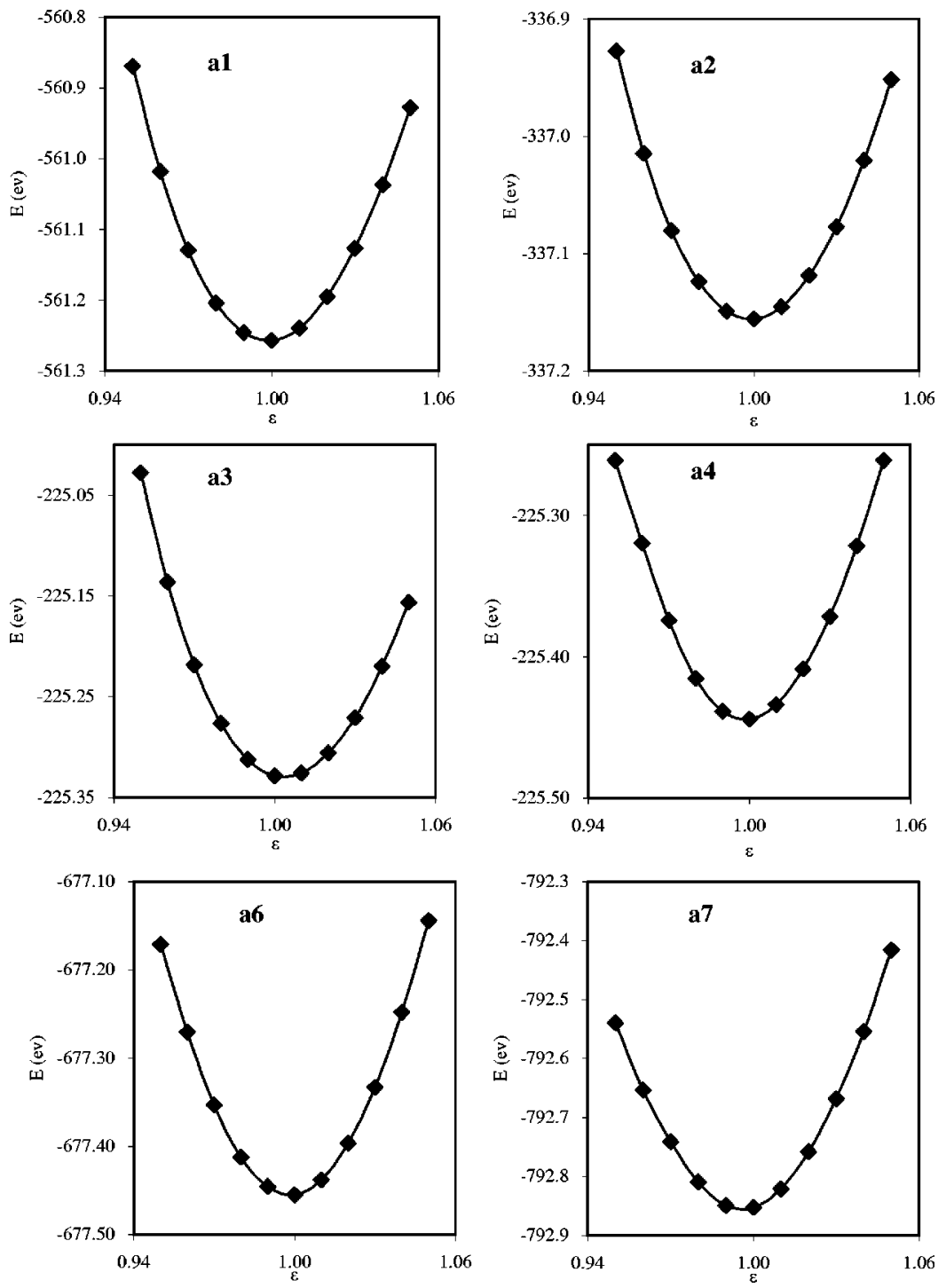

FIG. 4. Total energy of each structure in elongation and shortening. 

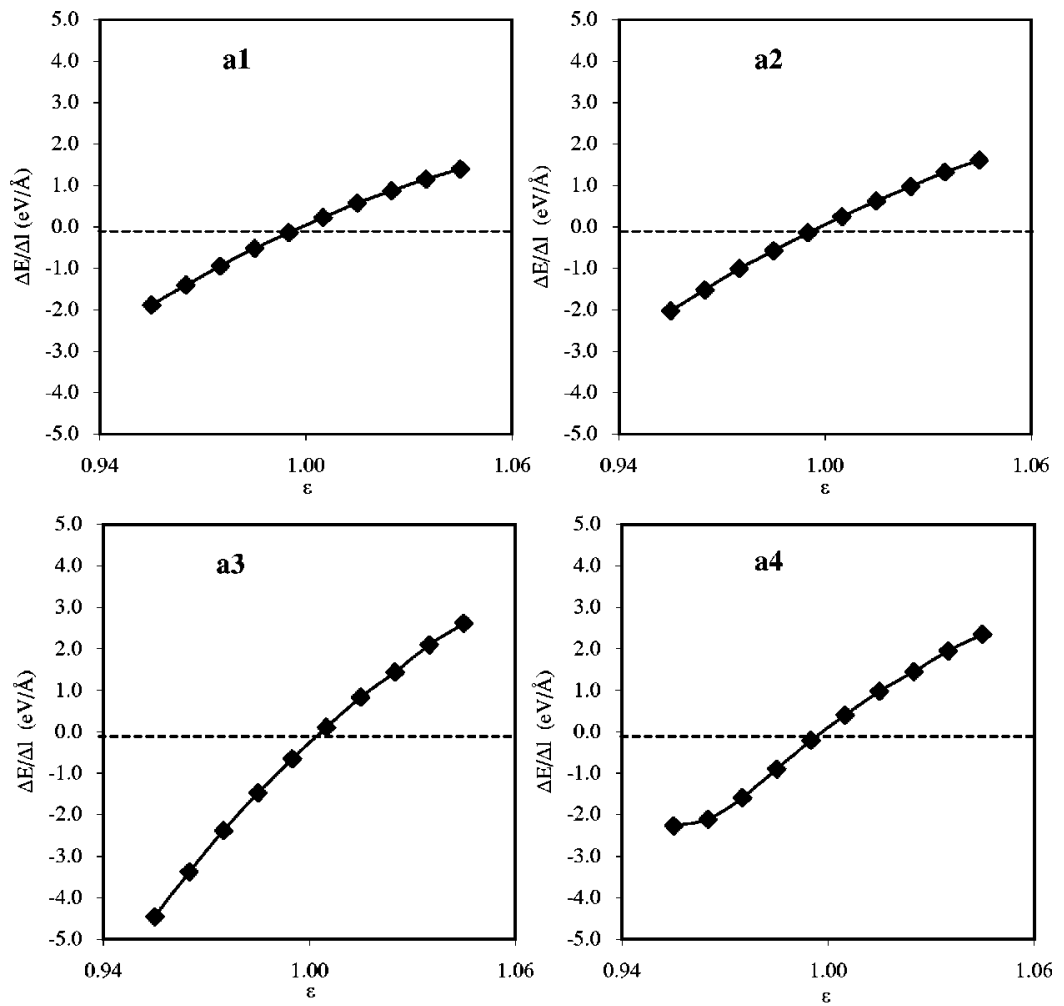

FIG. 5. $\Delta E / \Delta l$ of each structure in elongation and shortening.
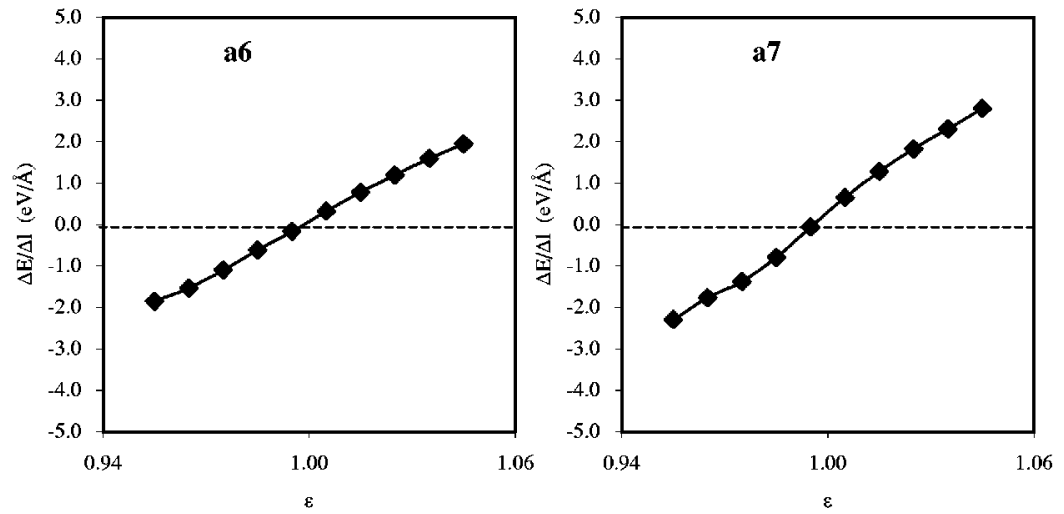

The models a6 and a7 represent coaxial nanowires with pentagonal and hexagonal rings, respectively. In these structures, the ring layers with constant wire radius, $r_{\mathrm{a} 6}$ or $r_{\mathrm{a} 7}$, and monoatom layers on axis are piled up alternately with constant interlayer distance, $t_{\mathrm{a} 6}$ or $t_{\mathrm{a} 7}$. They are also called the "icosahedral" type, because of a structural similarity with an icosahedron.

Each unit cell of the periodic boundary nanowire models for calculation is set up with the rectangular box of $8.361 \AA \times 8.361 \AA \times l_{\mathrm{a} i}$, that is, the edge lengths perpendicular to the wire are fixed at $8.361 \AA$, and the edge length parallel to the wire, the cell parameter $l_{\mathrm{a} i}$, is changed according to the structural optimization, which is equal to $10 t_{\mathrm{a} i}$ for $\mathbf{a 1}, 2 t_{\mathrm{a} i}$ for $\mathbf{a} 2-\mathbf{a} 4$, and $4 t_{\mathrm{a} i}$ for $\mathbf{a} 6$ and $\mathbf{a}$, respectively. The coordinates of $\mathrm{Al}$ atoms in each nanowire model are listed in Table I. We have carried out the structural optimization with keeping their symmetries by determining the parameters $r_{\mathrm{a} i}$ and $t_{\mathrm{a} i}$, which reduce the energy to the minimum. These parameters and interatomic distances in optimized structures are shown in Table II.

The structural optimization with keeping symmetry is in danger of obtaining meta-stable structures. Here, we have confirmed that our restrictions on symmetry for each model do not prevent us from finding the most stable structure of an isolated nanowire system by means of calculating normal vibrational modes for each model. Normal modes in a unit cell consist of three translational modes, one rotational mode with respect to the nanowire axis, and $(3 N-4)$ vibrational modes with $N$ being the number of atoms in a unit cell, from the viewpoint of one-dimensional periodic boundary. The stability of nanowire model was investigated by the deformation along the normal vibrational modes, where the norm of the eigenvectors was set to $0.05 \AA$. For some in-plane vibrational modes with $\Delta z=0$, particularly in a7 as shown in Fig. 2 , our structures are not stable against the deformation in the unit cell of $8.361 \AA \times 8.361 \AA \times l_{\text {ai }}$, because the interaction with other nanowires in neighboring cells is very large. However, all vibrational modes of deformation show stability against the deformation when the unit cell is enlarged as $14.817 \AA \times 14.817 \AA \times l_{\mathrm{a} i}$. Namely, our method of structural optimization gives the most stable structures for the isolated nanowire systems. Our results agree with the illustrations of 


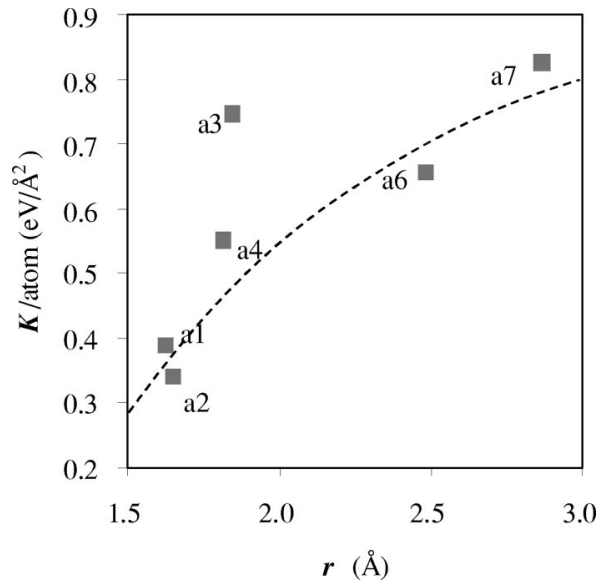

FIG. 6. Stabilities of structures against the elastic deformation $K$ with respect to the wire radius of each structure.

the most stable structures of $\mathrm{Al}$ nanowires in Ref. 4 using empirical potential functions.

The correlation between the energy per atom E/atom and the radius of nanowire $r$ is shown in Fig. 3. The radius of a4 was regarded as the average of $r_{\mathrm{a} 4}$ and $r_{\mathrm{a} 4}^{\prime}$. The shorter the radius of the nanowire is, the less stabilized the energy per atom has a tendency to be. When the radius of the nanowire becomes small, the structure changes to "weird wire" which does not contain any axial atoms such as a1-a4. As a result, the rate of surface atoms gets larger compared with the internal atoms, and, then, the effect of the surface energy surpasses that of the bulk cohesive energy.

\section{B. Stabilities against the elastic deformation}

We have discussed the stabilities of nanowires by calculating the energy conserved in them when they are elastically deformed along the direction of the axis. By using the same means as the structural optimization of nanowire models, each type of nanowire model has been elongated or contracted.

Figure 4 shows the energies of each model, where the cell parameter is set to $\varepsilon l_{\text {ai }, 0}(\varepsilon=0.95,0.96,0.97, \ldots, 1.04$, 1.05 ) with the optimized cell parameter $l_{\mathrm{a} i, 0}$. Each curve of energy $E$ with respect to $\varepsilon$ seems to be a parabola, and, certainly, $\Delta E / \Delta l$ becomes almost linear for each model as shown in Fig. 5. Now we can define an index of the stability of nanowires against the elastic deformation as

$$
K=\Delta^{2} E / \Delta l^{2} .
$$

The elastic stabilities per atom for $\varepsilon>0$ are shown in Fig. 6 . The figure indicates that the larger the radius of nanowire gets, the more reinforced its stability has a tendency to be. It is also found that structures with small cell parameter have good stability. These results represent the close correlation between the stability against the elastic deformation and that of the static energy. It is found that the effect of the bulk cohesive energy exceeds that of the surface energy in nanowire with small cell parameter.

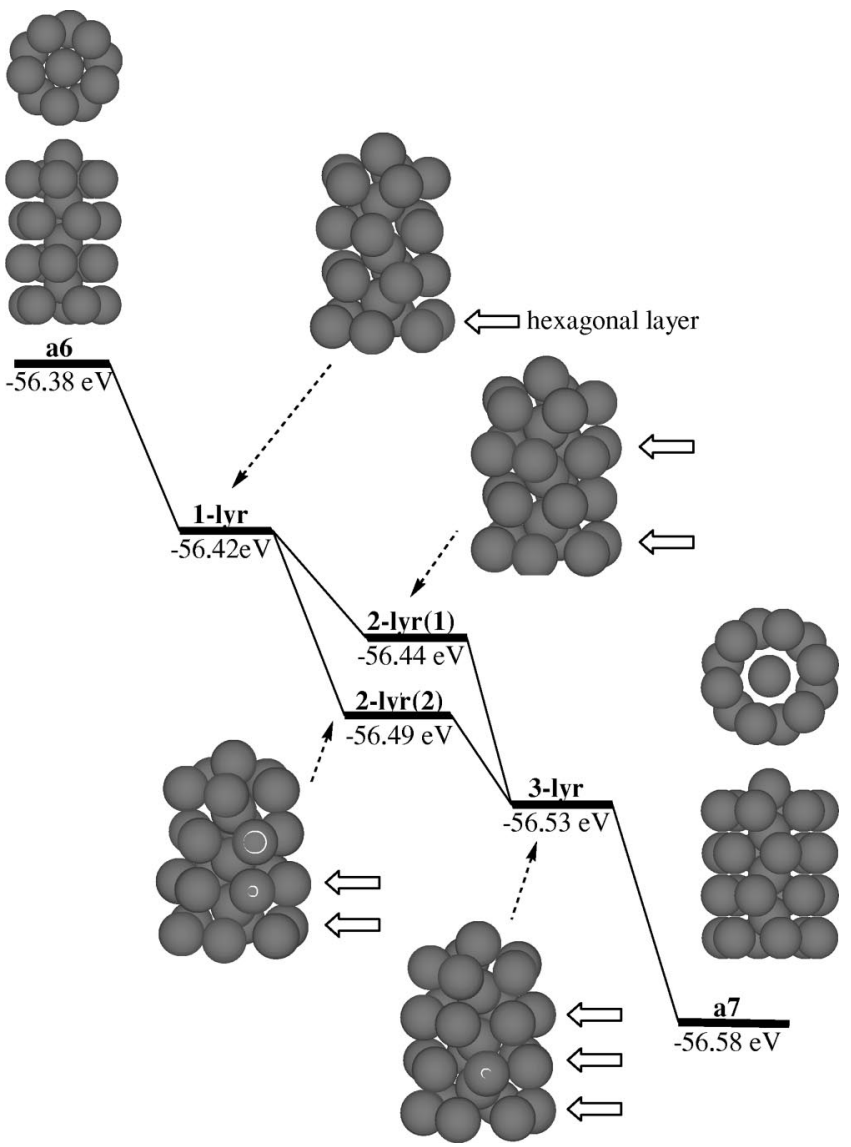

FIG. 7. Growing process from $\mathbf{a} 6$ to $\mathbf{a} 7$ and the energy per atom in each stage.

\section{Adsorption of an Al atom and growth of the coaxial nanowire}

Molecular beam epitaxy (MBE) and sputtering have been used for the methods of growing metal. In the growing process of $\mathrm{Al}$ nanowires, it is expected that the structure will be changed to that of a different type. As mentioned above, the energy of $\mathbf{a} 7$, which consists of hexagonal layers, is more stabilized than that of $\mathbf{a 6}$, which consists of pentagonal layers, for the coaxial nanowire models. This suggests the probability that a6 will be changed to a7 exothermally through the adsorption of $\mathrm{Al}$ atoms. Here, we simulated the growing process of a6 type $\mathrm{Al}$ nanowire to $\mathbf{a} 7$.

In this simulation, four pentagonal layers and for monoatom layers are contained in the unit cell. The edge lengths of unit cell are set to $8.731 \times 8.731 \times 10.08 \AA^{3}$. We assumed the growing process of an $\mathbf{a} 6$ type structure as follows

$$
\text { a6 } \rightarrow 1-\operatorname{lyr} \rightarrow(2-\operatorname{lyr}(1), 2-\operatorname{lyr}(2)) \rightarrow 3-\operatorname{lyr} \rightarrow \mathbf{a} 7,
$$

where 1-lyr, 2-lyr, and 3-lyr respectively denote one, two, and three of four pentagonal layers in $\mathbf{a 6}$ are replaced by hexagonal layers, as shown in Fig. 7. It is found that the energy per atom in each state is gradually stabilized. If no energy barrier exists in each step, the growing process to a7 proceeds rapidly. We discussed the change of energy and the reconstruction in the transition from a pentagonal ring to a hexagonal one, in the step from a6 to 1-lyr as an example. 


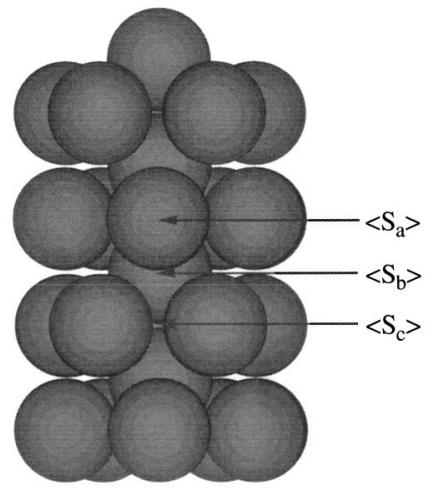

(a)

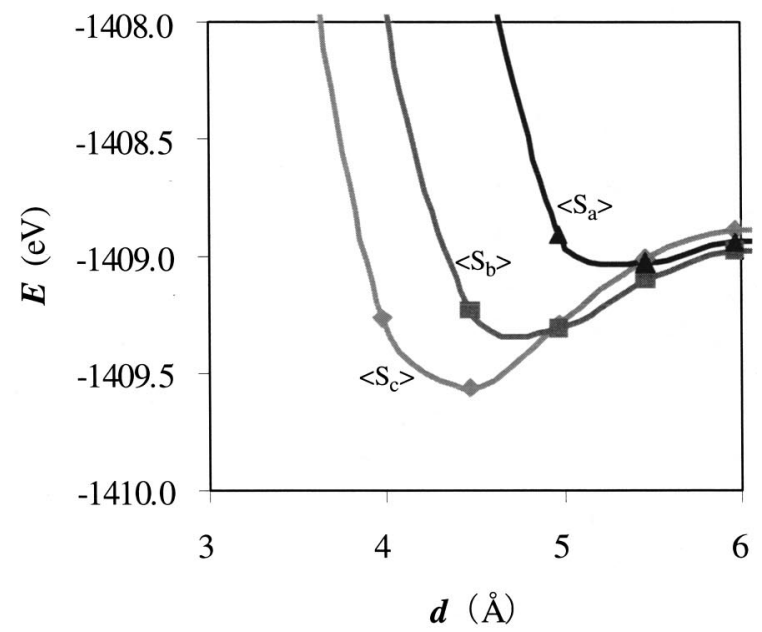

(b)

FIG. 8. (a) Adsorption spots $\left\langle S_{a}\right\rangle,\left\langle S_{b}\right\rangle$, and $\left\langle S_{c}\right\rangle$ for $\mathbf{a 6}$ and (b) adsorption energy curves by approaching an Al adatom on $\left\langle S_{a}\right\rangle,\left\langle S_{b}\right\rangle$, and $\left\langle S_{c}\right\rangle$ with respect to distance from the axis.

In order to specify where an $\mathrm{Al}$ atom adsorbed, we brought it to the spots $\left\langle S_{a}\right\rangle$ on the shell atom, $\left\langle S_{b}\right\rangle$ on the axis atom, and $\left\langle S_{c}\right\rangle$ between the shell atoms. Figure 8 shows the adsorption energy curves on these spots with respect to distance from the axis. We found that the adatom is most stabilized on the $\left\langle S_{c}\right\rangle$ about $4.5 \AA$ away from the axis.

When the $\mathrm{Al}$ adatom is put closer to the axis, the pentagonal ring is repelled by the adatom and reconstructed in the direction of the stabilization. The reconstruction by the approach of an $\mathrm{Al}$ adatom was observed as shown in Fig. 9,

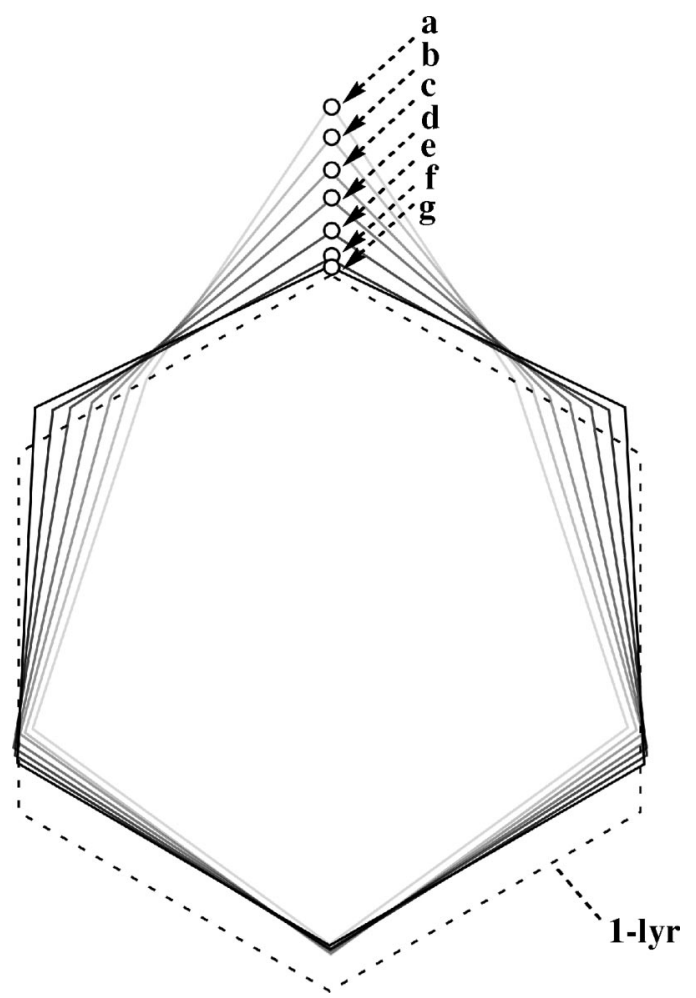

FIG. 9. Process of atom reconstruction from a pentagonal layer to a hexagonal one in the course from a6 to 1-lyr. Symbols a-g denote the position of the $\mathrm{Al}$ adatom. where we brought an adatom $0.25 \AA$ closer to the axis in every step. In order to simplify the calculation, we fixed the motion of all atoms except for five atoms of the pentagonal layer and two axis atoms next to it, and we restricted the motion of the seven atoms only on the plane perpendicular to the axis. It is found that the pentagonal ring eventually changes to the almost equilateral hexagonal one. Figure 10 shows the change of energy per atom in each stage along the reconstructing process from $\mathbf{a} 6$ to 1 -lyr. It revealed that there is no energy barrier in the reconstruction process. Accordingly, it is clarified that the pentagonal ring changes easily to the hexagonal one after an $\mathrm{Al}$ atom has been adsorbed.

In this article, we have demonstrated the electronic interaction between the adsorption atom and the pentagonal ring in terms of the quantum mechanical densities ${ }^{5-8}$ based on the regional density functional theory. ${ }^{5-12}$ The electronic kinetic energy density $n_{T}(\vec{r})$ is defined as ${ }^{5-8}$

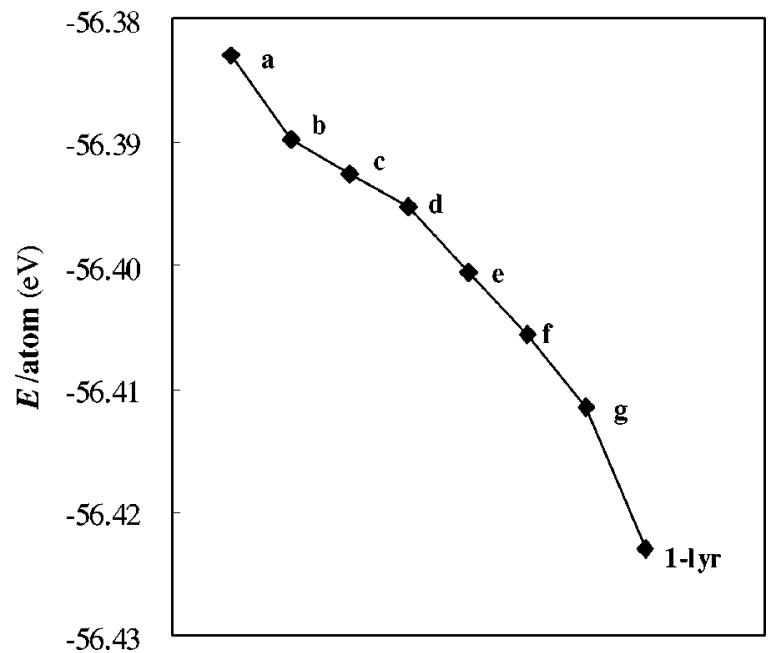

FIG. 10. Energy per atom along the reconstructing process from a6 to 1-lyr. Symbols a-g denote the stage where the $\mathrm{Al}$ adatom is located on the position as shown in Fig. 8. 


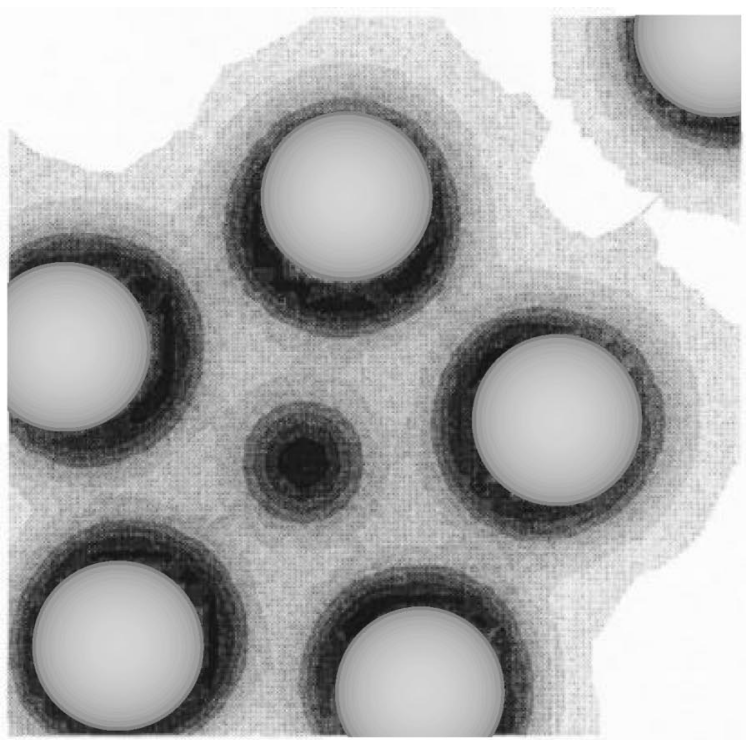

(a)

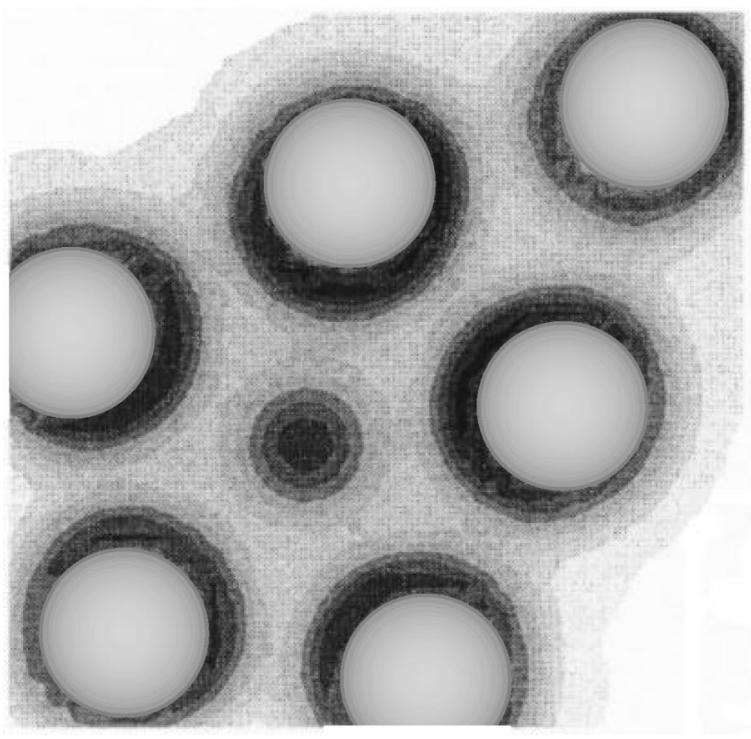

(b)

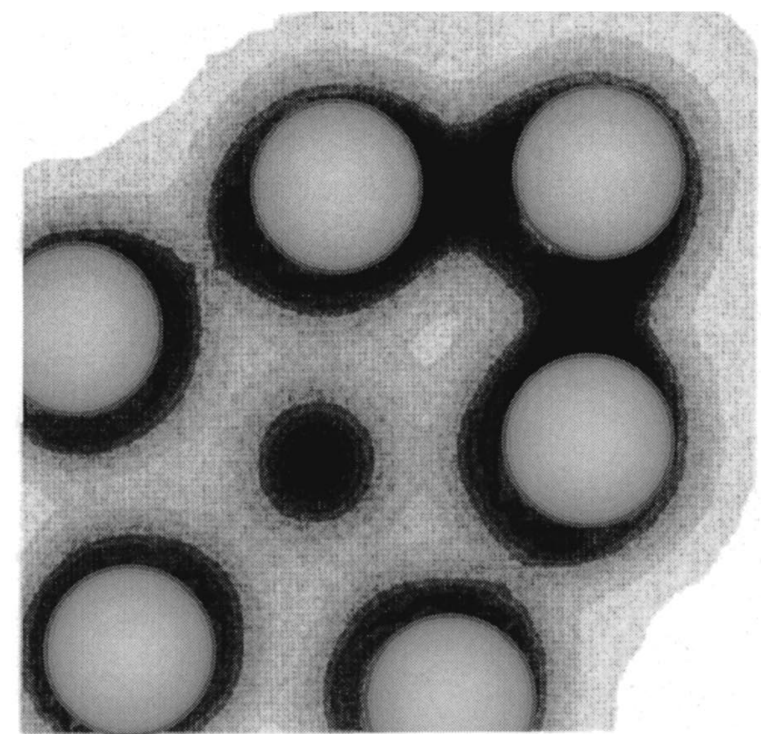

(c)

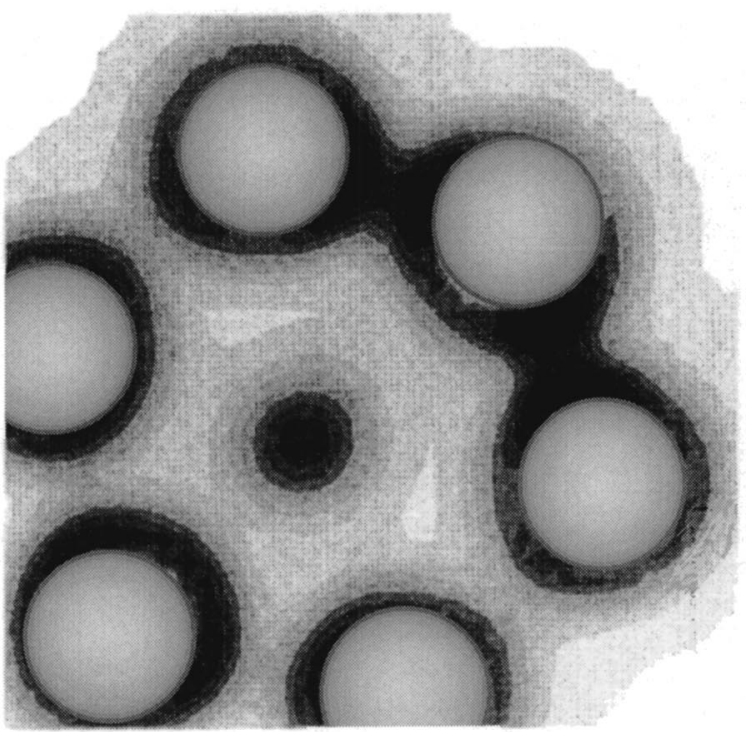

(d)

FIG. 11. Maps of $n_{T}(\vec{r})$ on the plane with the $\mathrm{Al}$ adatom where the distance from the axis is (a) $5.76 \AA$, (b) $4.60 \AA$, (c) $3.70 \AA$, and (d) $2.86 \AA$. Gray area denotes $R_{D}$. Circles on $\mathrm{Al}$ atoms represent a cross section of pseudopotential with the plane of map.

$$
\begin{aligned}
n_{T}(\vec{r})= & \frac{1}{2} \sum_{i} \nu_{i}\left(\left\{-\frac{\hbar^{2}}{2 m} \Delta \psi_{i}^{*}(\vec{r})\right\} \psi_{i}(\vec{r})\right. \\
& \left.+\psi_{i}^{*}(\vec{r})\left\{-\frac{\hbar^{2}}{2 m} \Delta \psi_{i}(\vec{r})\right\}\right),
\end{aligned}
$$

where $m$ is the mass of electron, $\psi_{i}(\vec{r})$ is the natural orbitals, and $\nu_{i}$ is the occupation number of $\psi_{i}(\vec{r})$, respectively. The region with $n_{T}(\vec{r})>0$ can be regarded as an area where electrons can move freely in the meaning of classical mechanics, called the electronic drop region $R_{D}$, and the region with $n_{T}(\vec{r})<0$ means an area where electrons can move only with the quantum mechanical tunneling effect, called the electronic atmosphere region $R_{a} . R_{D}$ and $R_{a}$ are divided with the electronic interface, the hypersurface of $n_{T}(\vec{r})=0 .^{5-8}$ The total electronic force density $\vec{F}^{S}(\vec{r})$ is represented as

$$
\vec{F}^{S}(\vec{r})=\vec{\tau}^{S}(\vec{r})+\vec{X}^{S}(\vec{r}),
$$

where $\vec{\tau}^{S}(\vec{r})$ and $\vec{X}^{S}(\vec{r})$ denotes the electronic tension density and the electronic external force density, respectively. The superscript $S$ means that these densities originate in 
the velocity density operator $\hat{S}(\vec{r}) .^{5-8}$ The $\vec{\tau}^{S}(\vec{r})$ has quantum mechanical origin, and is defined as $\vec{\tau} S(\vec{r})$ $={ }^{t}\left(\tau^{S 1}(\vec{r}), \tau^{S 2}(\vec{r}), \tau^{S 3}(\vec{r})\right)$ with

$$
\begin{aligned}
\tau^{S k}(\vec{r})= & \frac{\hbar^{2}}{4 m} \sum_{i} \nu_{i}\left(\psi_{i}^{*}(\vec{r}) \frac{\partial \Delta \psi_{i}(\vec{r})}{\partial x^{k}}-\frac{\partial \psi_{i}^{*}(\vec{r})}{\partial x^{k}} \Delta \psi_{i}(\vec{r})\right. \\
& \left.+\frac{\partial \Delta \psi_{i}^{*}(\vec{r})}{\partial x^{k}} \psi_{i}(\vec{r})-\Delta \psi_{i}^{*}(\vec{r}) \frac{\partial \psi_{i}(\vec{r})}{\partial x^{k}}\right)
\end{aligned}
$$

for $k=1,2,3$. The $\vec{X}^{S}(\vec{r})$ is defined in Eq. (13) in Ref. 5. In the stationary state, the $\vec{\tau}^{S}(\vec{r})$ exactly cancels the $\vec{X}^{S}(\vec{r}),{ }^{5-8}$ that is, $\vec{E}(\vec{r})$,

$$
0=\vec{\tau}^{S}(\vec{r})+\vec{X}^{S}(\vec{r})
$$

and the $\vec{X}^{S}(\vec{r})$ is equivalent to the electric field $\vec{E}(\vec{r})$ exerted on electron. ${ }^{6,7}$ As a result, the $\vec{E}(\vec{r})$ due to interaction between electrons can be visualized properly by means of $\vec{\tau}^{S}(\vec{r}) .^{5-8}$ Figures 11 and 12 display $n_{T}(\vec{r})$ and $\vec{\tau}^{S}(\vec{r})$ in the growing process of $\mathbf{a} 6$ nanowire. Circles on $\mathrm{Al}$ atoms represent a cross section of pseudopotential with the plane of map, and a core-orthogonality hole contribution due to the pseudopotential is ineffective out of the circles. In Fig. 11(a) where the distance from the axis is $5.76 \AA, R_{D}$ 's due to the $\mathrm{Al}$ adatom and the nanowire are separated from each other. It means that the electron redistribution is sluggish because the classical electron transfer cannot be expected. On the other hand, we can confirm that the $\mathrm{Al}$ adatom makes a large electronic interaction with the pentagonal ring by observing the connection of $R_{D}$ 's in Figs. 11(b)-11(d). Furthermore, in terms of $\vec{\tau}^{S}(\vec{r})$, it is found that the Al adatom interacts extensively only with two neighboring pentagonal-ring atoms where the distance from the axis is $3.70 \AA$ as shown in Fig. 12(a), but the $\mathrm{Al}$ adatom gets to interact with the axial Al atom in addition to the neighboring atoms where the distance from the axis is $2.86 \AA$ as shown in Fig. 12(b). Thus, the $\vec{\tau}^{S}(\vec{r})$ gives new images of microscopic electronic stresses.

\section{CONCLUSIONS}

We have carried out first-principle calculations for $\mathrm{Al}$ nanowires, and have determined stable structures for six types. It is observed that the energy per atom is raised as the radius of nanowire gets shorter. It means that increase of surface atoms reduces the effect of the bulk cohesive energy. We have investigated the stability of nanowires against the elastic deformation by introducing an index $K$ as defined in Eq. (1) and have shown that the stability against the elastic deformation is reinforced as the radius gets larger. This result indicates that the close correlation between the stability against deformation and that of the static energy.

We have also simulated the growing process of a pentagonal coaxial nanowire to a hexagonal one by adsorption of $\mathrm{Al}$ atom. We confirmed that the reconstruction from a pentagonal ring to a hexagonal one by an $\mathrm{Al}$ adatom proceeds exothermally without any energy barrier. Therefore, it is considered that the pentagonal coaxial nanowire changes easily to the hexagonal one after $\mathrm{Al}$ atoms have been adsorbed.

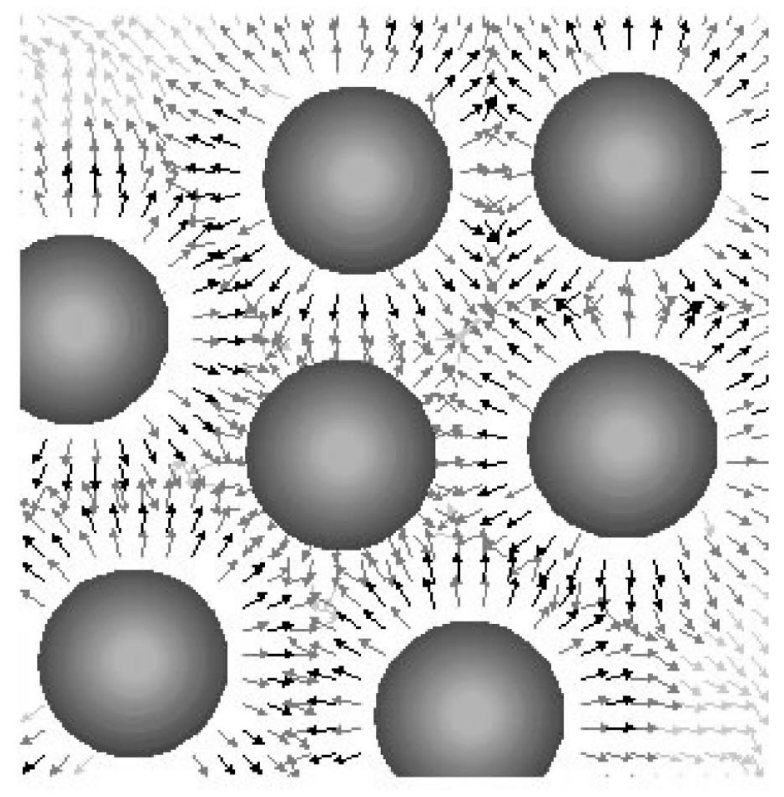

(a)

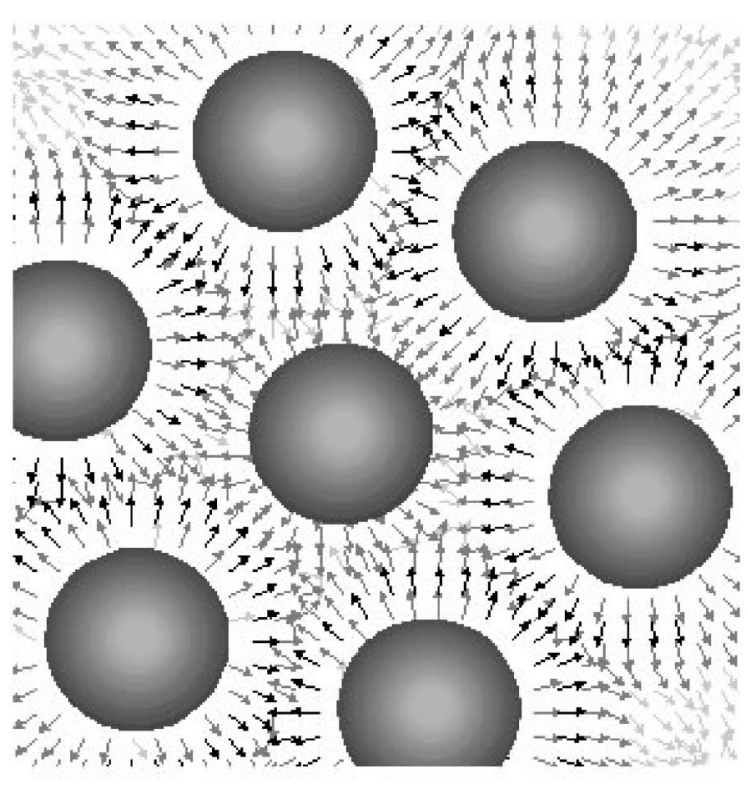

(b)

FIG. 12. Maps of $\vec{\tau}^{S}(\vec{r})$ on the plane with the $\mathrm{Al}$ adatom where the distance from the axis is (a) $3.70 \AA$ and (b) $2.86 \AA$. Circles on $\mathrm{Al}$ atoms represent a cross section of pseudopotential with the plane of map.

Along the reconstruction from a pentagonal ring to hexagonal one, the electronic processes and driving forces have been discussed in terms of the quantum mechanical energy densities $^{5-8}$ based on the regional density functional theory. ${ }^{5-12}$ The electronic kinetic energy density $n_{T}(\vec{r})$ and the electronic tension density $\vec{\tau}^{S}(\vec{r})$ have been applied to the local electronic nature in the reconstruction. They have disclosed the driving force of the stability in electronic processes and give new images of microscopic electronic stresses. 


\section{ACKNOWLEDGMENTS}

This work was supported by a Grant-in-Aid for Scientific Research from the Ministry of Education, Science and Culture of Japan, for which the authors express their gratitude..

${ }^{1}$ Y. Kondo and K. Takayanagi, Science 289, 606 (2000).

${ }^{2}$ V. Rodrigues, T. Fuhrer, and D. Ugarte, Phys. Rev. Lett. 85, 4124 (2000).

${ }^{3}$ A. Nakamura, M. Brandbyge, L. B. Hansen, and K. W. Jacobsen, Phys. Rev. Lett. 82, 1538 (1999).

${ }^{4}$ O. Gülseren, F. Ercolessi, and E. Tosatti, Phys. Rev. Lett. 80, 3775 (1998).

${ }^{5}$ A. Tachibana, J. Chem. Phys. 115, 3497 (2001).

${ }^{6}$ A. Tachibana, in Stress Induced Phenomena in Metallization, edited by S. P. Baker (American Institute of Physics, New York, 2002), pp. 105-116.

${ }^{7}$ A. Tachibana, in Fundamental Perspectives in Quantum Chemistry: A
Tribute to the Memory of Per-Olov Löwdin, edited by E. Brandas and E. Kryachko (Kluwer Academic, Dordrecht, 2003).

${ }^{8}$ A. Tachibana, in Reviews in Modern Quantum Chemistry: A Celebration of the Contributions of Robert Parr, edited by K. D. Sen (World Scientific, Singapore, 2002), Chap. 45, pp. 1327-1366.

${ }^{9}$ A. Tachibana, Int. J. Quantum Chem., Quantum Chem. Symp. 21, 181 (1987).

${ }^{10}$ A. Tachibana and R. G. Parr, Int. J. Quantum Chem. 41, 527 (1992).

${ }^{11}$ A. Tachibana, Int. J. Quantum Chem. 57, 423 (1996).

${ }^{12}$ A. Tachibana, Theor. Chem. Acc. 102, 188 (1999).

${ }^{13}$ Periodic Regional DFT program package, ver. 1, Tachibana Lab., Kyoto University, Kyoto, 2001.

${ }^{14}$ D. R. Hamann, Phys. Rev. B 40, 2980 (1989).

${ }^{15}$ J. P. Perdew and Y. Wang, Phys. Rev. B 45, 13244 (1992).

${ }^{16}$ J. P. Perdew, J. A. Chevary, S. H. Vosko, K. A. Jackson, M. A. Pederson, D. J. Singh, and C. Fiolhais, Phys. Rev. B 46, 6671 (1992). 